\title{
Self-assembling, protein-based intracellular bacterial organelles: emerging vehicles for encapsulating, targeting and delivering therapeutical cargoes
}

\author{
José L Corchero ${ }^{1,2,4^{*}}$ and Juan Cedano 2,3
}

\begin{abstract}
Many bacterial species contain intracellular nano- and micro-compartments consisting of self-assembling proteins that form protein-only shells. These structures are built up by combinations of a reduced number of repeated elements, from 60 repeated copies of one unique structural element self-assembled in encapsulins of $24 \mathrm{~nm}$ to 10,000-20,000 copies of a few protein species assembled in a organelle of around 100-150 nm in cross-section. However, this apparent simplicity does not correspond to the structural and functional sophistication of some of these organelles. They package, by not yet definitely solved mechanisms, one or more enzymes involved in specific metabolic pathways, confining such reactions and sequestering or increasing the inner concentration of unstable, toxics or volatile intermediate metabolites. From a biotechnological point of view, we can use the self assembling properties of these particles for directing shell assembling and enzyme packaging, mimicking nature to design new applications in biotechnology. Upon appropriate engineering of the building blocks, they could act as a new family of self-assembled, protein-based vehicles in Nanomedicine to encapsulate, target and deliver therapeutic cargoes to specific cell types and/or tissues. This would provide a new, intriguing platform of microbial origin for drug delivery.
\end{abstract}

Keywords: self-assembling proteins, protein delivery, nanoparticles

\section{Introduction}

Our view of the intracellular bacterial cell organization has significantly changed over the past years. Once taken simply as reaction vessels containing a homogeneous solution of proteins, bacteria are now seen as organisms with an intricate subcellular architecture in which individual proteins localize to particular sites within the cell, often in a dynamic manner [1-4]. Protein-based microcompartments are particularly intriguing intracellular bacterial organelles. They are large macromolecular complexes consisting of metabolic enzymes encapsulated within multiprotein, polyhedral shells, reminiscent of the viral capsids structures. A common feature of such bacterial microcompartments

\footnotetext{
* Correspondence: jlcorchero@ciber-bbn.es

${ }^{1}$ CIBER de Bioingeniería, Biomateriales y Nanomedicina (CIBER-BBN)

Barcelona, Spain

Full list of author information is available at the end of the article
}

(BMC) is a thin shell primarily (composed by a few thousand protein subunits, so-called BMC shell proteins) that encapsulate the enzymes while allowing transport of substrates and products. Several studies indicate that the general role of the protein shell is to seclude toxic or volatile metabolic intermediates, while allowing enzyme substrates, products and cofactors to pass. Polyhedral organelles had been identified and visualized by electron microscopy in cyanobacteria and some chemoautotrophs, and were first isolated in 1973 and determined to contain the $\mathrm{CO}_{2}$-fixing enzyme RuBisCO $[5,6]$. They were named carboxysomes, and are now recognized as the first member of a diverse group of microcompartments. BMC proteins were later found to be encoded in the propanediol utilization operon ( $p d u$ operon) of the heterotroph Salmonella [7] and by an operon for metabolizing ethanolamine (eut operon) in enteric bacterial species, including

\section{() Biomed Central}


Salmonella and Escherichia [8]. Until recently, the diversity of these structures had been overlooked because many of them are not formed on standard growth media and because their observation requires the use of electronic microscopy techniques. However, recent genome analyses have detected seven functionally distinct organelles distributed among over at least 40 bacteria genera. Searches for homologies with shell proteins in protein sequence databases have emphasized the widespread occurrence of microcompartments across bacteria species, and the likely horizontal gene transfer for their genetic determinants spread [9].

\section{Main polyhedral organelles in bacteria Carboxysomes}

Isolated in the early 1970s, carboxysomes were the first bacterial polyhedral organelle identified $[5,6]$. They are polyhedral inclusions of approximately $100-150 \mathrm{~nm}$ in cross section, and with a 3-4 $\mathrm{nm}$ protein shell composed of six to ten different protein species. These organelles have been identified in cyanobacteria and in many chemoautotrophic bacteria, but not in eukaryotes [10-12]. Their distribution and composition has been investigated by genomic and phylogenetic analyses [13]. Their structure varies among different producing bacteria, and according to their constituent proteins, they can be divided into subtypes $\alpha$ and $\beta$. The $\alpha$-type carboxysome is that of the facultative chemoautotroph Halothiobacillus neapolitanus. Type $\beta$ carboxysomes are found in the $\beta$ subdivision of the cyanobacteria, where carboxysome proteins are designated CCM because of their role in a carbon dioxide concentrating mechanism. The function of carboxysomes is to enhance autotrophic $\mathrm{CO}_{2}$ fixation at low $\mathrm{CO}_{2}$ levels. This role is supported by the findings that carboxysome formation is induced by $\mathrm{CO}_{2}$ limitation [14], and that mutant strains of cyanobacteria and chemoautotrophs unable to properly form carboxysomes require high $\mathrm{CO}_{2}$ levels for autotrophic growth $[15,16]$. The carboxysome is filled with the ribulose bisphosphate carboxylase/oxygenase (RuBisCO) enzyme [6], which catalyzes the $\mathrm{CO}_{2}$ fixation step in the Calvin cycle. Also associated with the carboxysome is the carbonic anhydrase (CA) enzyme, responsible of the conversion of $\mathrm{HCO}_{3}^{-}$(that is not used by RuBisCO) to $\mathrm{CO}_{2}$, the substrate for RuBisCO [17-19].

\section{Organelles for 1,2-propanediol utilization ( $p d u$ )}

Carboxysomes were the only known polyhedral organelles for many years. However, Salmonella enterica was found to form a polyhedral organelle during growth on 1,2-propanediol (1,2-PD) as a sole carbon and energy source [20]. Electron microscopy showed that S. enterica forms structures (similar in size and shape to carboxysomes) during growth on 1,2-PD but not during growth on other carbon sources [20,21]. Microscopy analysis showed that coenzyme B12-dependent diol dehydratase (DDH) is a major component of the pdu organelles, and that the PduA protein is a shell component $[20,22]$, together with other 14 different polypeptides. Genetic studies showed that genes specifically involved in 1,2-PD utilization encode homologs of the carboxysome shell proteins [7], but that their enzyme cargo and physiological functions are clearly different [21-23]. The pdu organelles function is to minimize the harmful effects of a toxic intermediate of 1,2-PD degradation (propionaldehyde) [21-23]. Mutants unable to form pdu organelles undergo a 20-h period of growth arrest during degradation of 1,2-PD, whereas wild-type $S$. enterica grows normally under similar conditions [21]. The length and severity of growth arrest increases at higher 1,2-PD concentrations, suggesting that it results from the accumulation of a toxic metabolite derived from 1,2-PD [21].

\section{Organelles involved in coenzyme B12-dependent ethanolamine degradation (eut)}

It has also been described a polyhedral organelle involved in ethanolamine utilization (eut operon) by $S$. enterica [8]. The Eut and Pdu microcompartments share some homologous enzymes: both metabolic pathways proceed via aldehyde intermediates, propionaldehyde in the case of Pdu and acetaldehyde in the case of Eut [2]. The Eut microcompartment function is to metabolize ethanolamine without allowing the release of acetaldehyde into the cytosol, thus alleviating the potentially toxic effects of excess aldehyde in the bacterial cytosol [24-26] and also preventing volatile acetaldehyde from diffusing across cell membrane and leading to a loss of carbon [27]. The initial step of this process is catalyzed by coenzyme B12-dependent ethanolamine ammonia lyase, which converts ethanolamine to acetaldehyde. Subsequently, acetaldehyde is converted to ethanol and acetate by a series of reactions analogous to those used for 1,2-PD degradation [28]. This suggests that Eut and Pdu organelles might have the common function of protecting cells against aldehyde toxicity [29]. In addition, recent studies (referring to the Eut organelles as metabolosomes) suggest that these structures function concentrating both enzymes and their substrates to allow a more efficient growth while minimizing acetaldehyde toxicity and maintaining coenzyme A balance [30].

\section{BMC shell structure}

At present, about 1700 proteins containing BMC domains have been identified, covering at least 10 different bacterial phyla. Multiple paralogs of the shell proteins are essentially always found together. The typical BMC domain consists of 90 amino acids in length with an alpha/beta fold pattern $[9,31]$. BMC proteins self- 
assemble to form disc-shaped hexamers, the basic building blocks of the shell (Figure 1, light blue proteins). Each hexamer typically presents a narrow pore through the middle, along the six-fold axis of symmetry. Crystal structures show that such hexamers further assemble side-by-side, forming a molecular layer $[9,32,33]$, with 4to 6 - $\AA$-wide gaps between hexamers. Both central hexamer pores and the gaps between hexamers potentially serve as gates for metabolites, selectively allowing passage of negatively charged molecules such as the substrates and products of $\mathrm{RuBis} \mathrm{CO}$ while restricting uncharged molecules such as $\mathrm{CO}_{2}$ and $\mathrm{O}_{2}$ [9]. A notable feature of the hexagonal layer of the carboxysome shell is the presence of a bowl-shaped depression or concavity on one side of the hexameric building block, side where both $\mathrm{N}$-termini and $\mathrm{C}$-termini are usually located. This molecular layer appears to represent the flat facets of the shell.

Electron microscopy studies have confirmed that carboxysomes are approximately icosahedral in shape. The construction of large icosahedral structures typically requires a combination of hexameric and pentameric units. Pentamers generate curvature in an otherwise flat hexagonal sheet, occupying the vertices of the icosahedral shell. In agreement with this vision, homologous proteins CcmL and CsoS4A from two different types of carboxysomes self-assemble to form pentamers (Figure 1 , orange proteins), whose size and shape are compatible with their placement at the vertices of an icosahedral shell containing 12 pentamers [32]. Both CcmL and CsoS4A proteins were expressed in Escherichia coli and an atomic model was built and refined at a resolution of
$2.4 \AA$ and $2.15 \AA$ for CcmL and CsoS4A, respectively. The study of such crystal structures confirmed that both CcmL and OrfA formed symmetric pentamers [32]. This model of $\mathrm{CcmL}$ or $\mathrm{CsoS} 4$ pentamers occupying the icosahedral vertices is in agreement with their low abundance in the shell, and with mutational experiments in which deletion of the genes led to failure to form closed shells [34]. However, there is not a total consensus on the role of $\mathrm{CcmL}$ or $\mathrm{CsoS} 4$ proteins with respect to provide the needed curvature in order to form icosahedral, closed shells. Some studies [35] have shown that CsoS4 proteins are apparently not essential determinants of carboxysome shape: a Halothiobacillus neapolitanus knockout mutant that does not produce CsoS4 predominantly forms carboxysomes of normal appearance, in addition to some elongated microcompartments. However, and despite their normal shape, mutant carboxysomes are functionally impaired (in the absence of CsoS4 protein, the carboxysome shell loses its permeability to $\mathrm{CO}_{2}$ ).

This scenario of hexamers forming flat facets and pentamers providing the needed curvature in the overall icosahedral structure (as shown in Figure 1) is not universal for all types of bacterial microcompartments. For example, the homologous protein of $\mathrm{CcmL}$ and CsoS4 in the Eut microcompartment is EutN, whose structure is hexameric rather than pentameric. The difference between the oligomeric state of EutN, compared to $\mathrm{CcmL}$ and CsoS4, presumably reflects structural differences between the Eut microcompartment and the carboxysome. Another alternative is that the Eut microcompartment could lack pentamers, which would

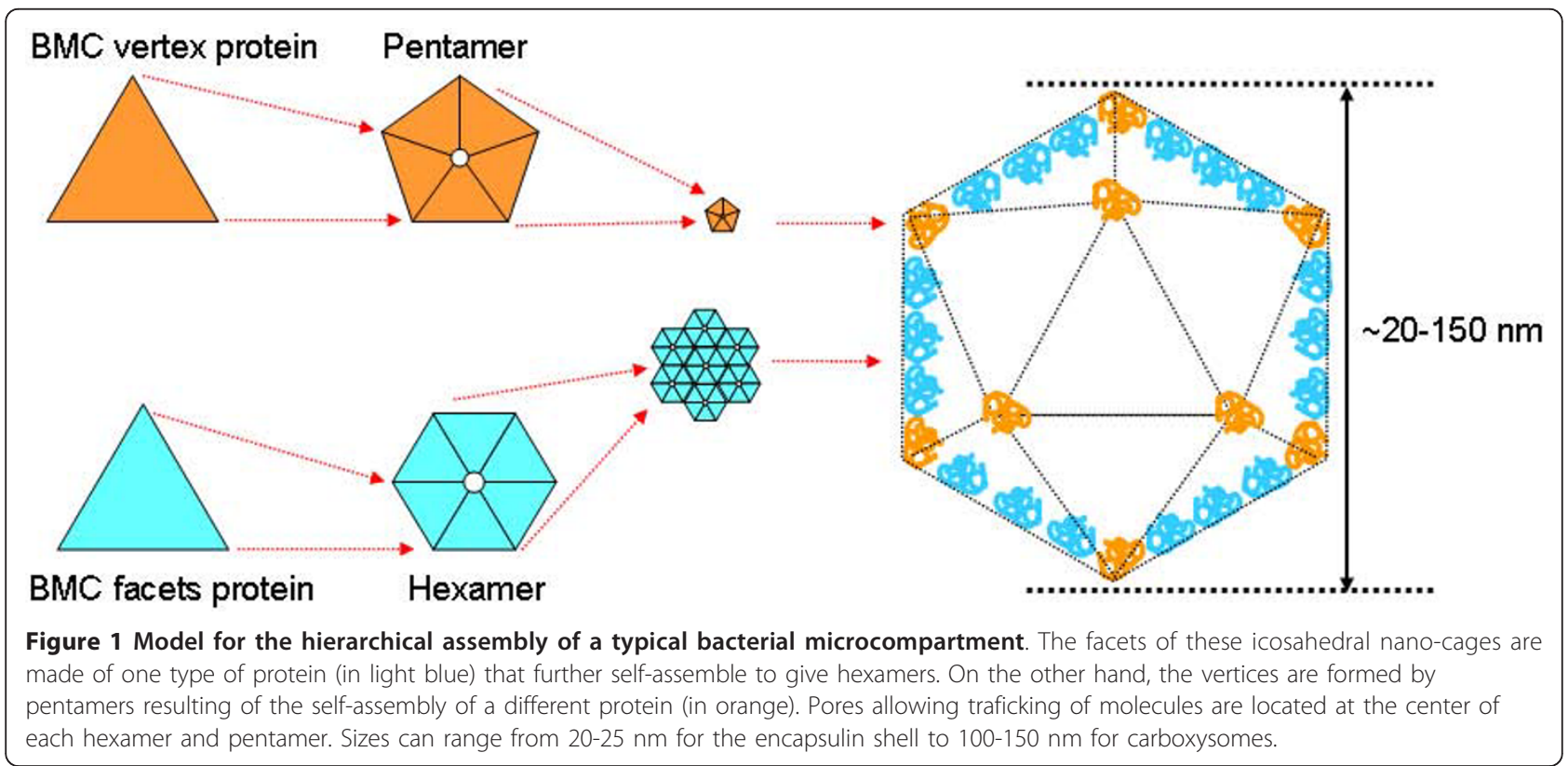


explain why enteric bacteria BMC tend to have less-regular icosahedral shapes than carboxysomes. Purified Pdu microcompartments contain two major BMC-domain proteins ( $\mathrm{PduA}$ and $\mathrm{PduJ}$ ) that are closely related in sequence to the hexamers proposed to form the faces of the carboxysome [22]. The $p d u$ operon also encodes a homolog $(\mathrm{PduN})$ of the pentamer proposed to from the vertices of the carboxysome [32]. These analogies suggest that Pdu shell may have flat faces formed by PduA and PduJ hexamers, and vertices made from PduN pentamers. However, and as seen for the Eut microcompartment, electron microscopy shows that Pdu microcompartments are more irregular in shape than carboxysomes: Pdu (and Eut) microcompartment does not resemble a regular icosahedron as closely as the carboxysome does, fact that may correlate with the different behaviors of the CcmL/CsoS4/EutN/PduN family of proteins.

\section{Encapsulating therapeutic proteins into protein-based bacterial organelles}

As the number of therapeutics requiring parenteral administration increases, delivery vehicles improving therapeutic efficacy will find increasing use [36-39]. Among all the currently available vehicles, the utility of nanoparticles to deliver chemotherapeutics in vivo is now well established, with several formulations and synthesis protocols available [40-42]. They can be synthesized from a range of materials, being the most common ones based on biocompatible, and preferably biodegradable, lipids or polymers [40]. In general, nanoparticles are designed to minimize limitations of conventional drug delivery systems, including nonspecific biodistribution and targeting, low aqueous solubility, poor bioavailability and low therapeutic effects due to insufficient drug concentration at disease sites. Moreover, nanoparticles of submicrometer size offers clear advantages over microparticles as they are best suited for intravenous delivery [43].

Macromolecular self-assembly has been exploited recently to engineer materials for the encapsulation and controlled delivery of therapeutics. Self-assembled structures can be formed by a variety of building blocks, both organic and inorganic [44]. Peptides and proteins are among the most useful organic building blocks, since they are stable and robust and can spontaneously associate to form nanotubes, nanospheres, nanofibrils, and other ordered nano-sized structures $[45,46]$. For example, since it was described that protein aggregation as bacterial inclusion bodies does not necessarily mean the inactivation of the forming proteins [47], such structures incorporated to the list of self-assembling materials with putative biotechnological and biomedical applications [48]. When protein self-assembling does not occur spontaneously, some strategies to promote it can be used. For example, fusion of self-assembling or arginine-rich peptides has been reported to promote the formation of active protein aggregates in E. coli $[49,50]$ or of structured nanodisks [51], respectively.

Protein-based capsids (also known as protein nanocages or nano-containers) are appealing vehicles for drug delivery [52,53]. Their biocompatibility, biological fabrication, functional diversity and versatility of design though protein engineering (assisted by in silico instruments) make them extremely powerful materials. A well-studied example of natural protein nano-cage is ferritin, a $450 \mathrm{kDa}$ protein assembly of 24 subunits. Ferritin, ubiquitous in cells and in extracellular matrices, is a cage-like protein with an inner space of $8 \mathrm{~nm}$ in diameter. This inner space allows storage of up to $4500 \mathrm{Fe}$ atoms as ferric oxyhydroxide clusters, serving as a reservoir providing Fe atoms for metabolic use [54]. Viral capsids, and other protein cages, are increasingly being used as multivalent, multifunctional nano-containers. Mimicking viral structures as models, several proteinonly nano-cages have been explored for materials applications [55].

Mechanisms directing enzyme encapsulation within protein-based bacterial organelles have been studied and revealed during the last years. In some cases (Figure 2, pannel A), a stretch of a few $(\sim 15-20)$ aminoacids at the $\mathrm{N}$-terminus of the inner cargo protein directs and binds it to specific sites on the inner surface of the shell protein. When such directing peptide is not present, the strategy is to synthesize the cargo protein together with the shell-forming domain from one unique gene (Figure 2, pannel B).

As an example of the first strategy (shown in Figure 2 , pannel $A$ ), in $\beta$ carboxysomes the protein $\mathrm{CcmM}$ is used as a scaffold to form interactions between both shell proteins and enzymes [56,57], through a CcmM C-terminal region with homology to the small subunit of RuBisCO [58], and probably mimicking the native interactions of small and large subunits of RuBisCO inside the carboxysome. In Pdu microcompartments some of the internal enzymes are also directed to the interior by special $\mathrm{N}$-terminal targeting sequences $[34,59]$. Fan and colleagues [59] demonstrated that a short N-terminal peptide is necessary and sufficient for packaging enzymes into the Pdu microcompartment. Deletion of 10 or 14 amino acids from the $\mathrm{N}$-terminus of the PduP enzyme (normally found within this microcompartment), significantly impaired its packaging, without affecting its enzymatic activity. On the other hand, fusion of the $18 \mathrm{~N}$-terminal amino acids from PduP to GFP, GST, or maltose-binding protein resulted in the encapsulation of these proteins within the Pdu microcompartment. It is worthy to note that 


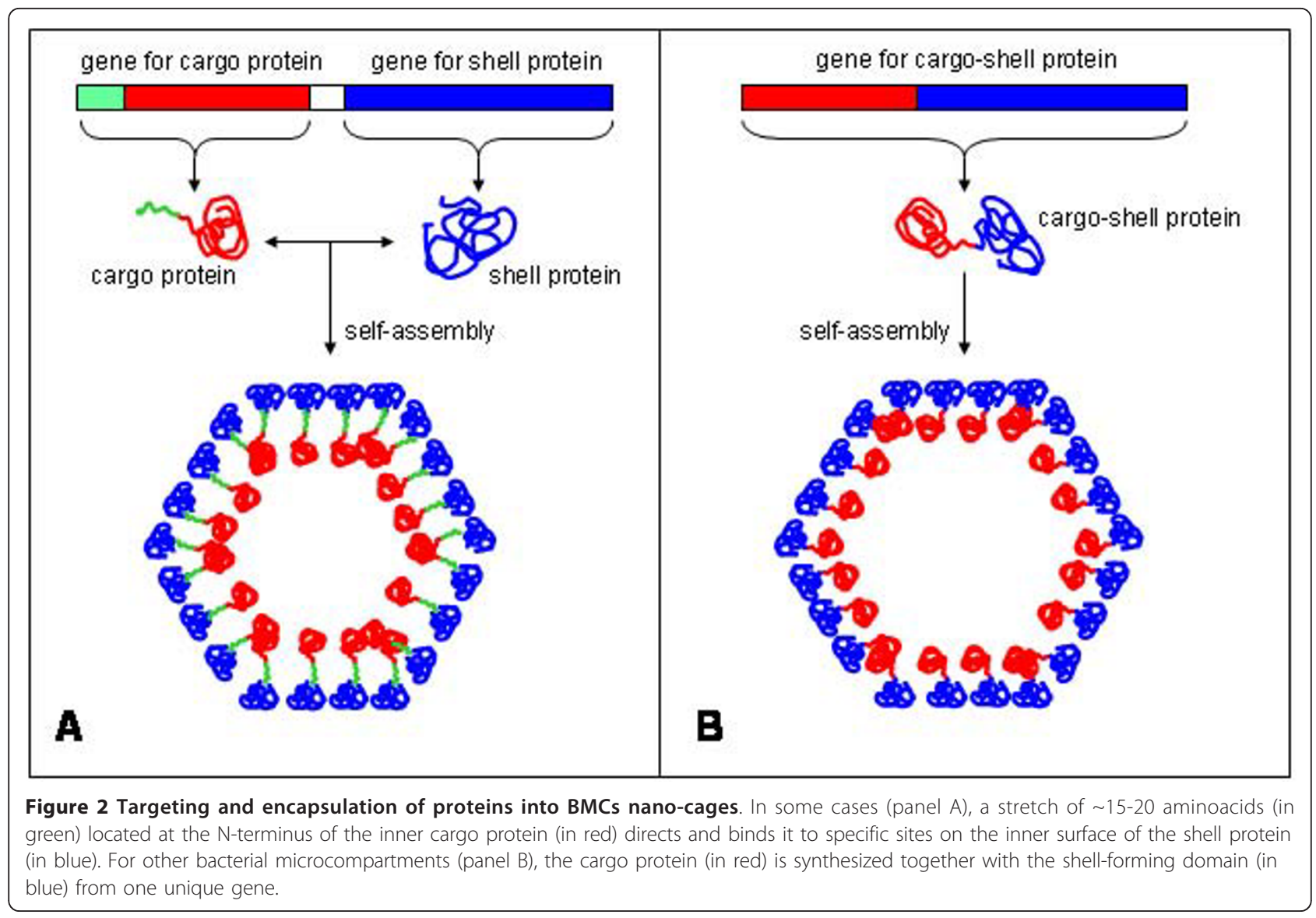

the Pdu shell can be assembled heterologously in the absence of native interior enzymes [34], and that carboxysomes can self-assemble in vivo when RuBisCO has been deleted [60], offering appealing opportunities to manipulate such nano-cages in the laboratory in order to fill them with therapeutic molecules. Also in this line, another landmark study about enzyme encapsulation was provided by Sutter and colleagues [61]. They described the smallest (20-24 nm of diameter) known protein-based organelle (found in the hyperthermophilic bacterium Thermotoga maritima). The protein family was initially named linocin [62], but it was renamed by the authors as "encapsulins". In many bacteria, the encapsulin gene is positioned downstream within an apparent two-gene operon, being preceded by the gene for either an iron-dependent peroxidase (DyP) or a protein closely related to the iron transporter ferritin (Flp). Sequence alignment of DyP and Flp genes revealed that only those followed by the encapsulin gene carry a $\mathrm{C}$-terminal extension with a conserved amino acids sequence responsible for the protein's physical interaction with the encapsulin protein, by binding to distinct pockets on the interior of the nanocompartment surface.
Support for the existence of the second strategy (Figure 2, pannel B) to encapsulate cargo proteins is provided by the hyperthermophilic archaeon Pyrococcus furiosus, where a Flp coding sequence (without any targeting sequence directing its encapsulation by physical interaction with BMC proteins) is fused in frame with an encapsulin gene [61]. In this situation, cargo and encapsulin proteins are synthesized as a fusion that further self-assembles to form a nano-cage containing the cargo protein.

Specific targeting sequences could be of use in biotechnological applications to package proteins inside the stable self-assembled icosahedral shell of encapsulin. As an example of the utility of such approach, an icosahedral enzyme complex, lumazine synthase, was engineered to encapsulate target molecules by means of charge complementarity. The lumazine synthase from Aquifex aeolicus (AaLS) represents the container component, as it forms icosahedral capsids large enough to encapsulate proteins. To engineer the charge environment within the capsid, four residues of each monomer that project into the lumen (Arg83, Thr86, Thr120, and Gln123) were mutated to glutamates, introducing extra negative charges to the inner surface of the protein 
cage. The hypothesis of the authors was that adding a short stretch of positively charged amino acids to a cargo protein should promote its specific encapsulation within this modified proteinaceous nano-container, as was demonstrated by using a modified GFP containing ten arginine residues [63].

\section{Use of protein-based bacterial organelles as drug delivery system}

Once the BMC is loaded with a therapeutic cargo protein, its administration would depend on the target to reach, adopting for each case the most appropriate way. A parenteral administration should be the most common choice to administrate this therapeutic agent, but not the only one. For mouth or throat cancer, oral administration would be a more appropriate method. On the other hand, to treat myeloblastomas the way would be the cerebrospinal fluid.

The field of cell-specific targeting has been significantly advanced, identifying targeting peptides that target their cargos to the vasculature of a variety of tissues, organs, and tumors $[64,65]$. It would be possible to add on the surface of BMC nanocages any type of specific binding domain, including targeting peptides or antibodies, providing the desired specificity. In this line, it was shown [66] that genetic addition of a targeting peptide (RGD-4C) or chemical conjugation of an anti-CD4 monoclonal antibody onto the surface of the small Hsp cage structure conferred specific cell targeting capacity. In addition, authors were able to load a cargo molecule, a fluorescent imaging agent (fluorescein), within the interior cavity of the Hsp cage, demonstrating the multifunctional capacity of protein nanocage architectures and their potential uses in medicine.

After reaching target cells, release of the cargo is another important issue for drug delivery. One strategy for particle disassembly and drug release is to take advantage of the increase in acidity once these macromolecules are taken up by the endocytotic pathway, where drug release would take place after BMC degradation in the lysosome [67]. In this line, it has been investigated the potential of using histidines for introducing $\mathrm{pH}$-dependent disassembly $[68,69]$. The results demonstrated that modulating the degree of electrostatic repulsion through changing the number of histidine interactions at subunit interfaces could be a generally applicable strategy for designing $\mathrm{pH}$-triggered assembly/ disassembly in protein macromolecular structures.

The use of BMC as a drug delivery system arises some general concerns, like its putative antigenicity. It could be modulated not only by engineering the exposed sequence/s of the constructs, but also by means of posttranscriptional or chemical modification (like pegylation [70]). Other materials used as a carriers (like Teflon, titanium, silicone oil, etc.) are not naturally processed by cells, sometimes remaining forever in the body of the patients and triggering unwanted reactions. On the contrary, due to its proteic nature, recycling by the cells of the BMC carrier would be relatively easy, only requiring the use of one of their multiple proteolytic pathways.

\section{Conclusions}

In protein-based therapies, there are several known issues to be carefully considered, like a short protein half-life in vivo, side effects caused by the multiple or high doses that must be administered in order to reach the desirable concentration in the cell, or possible protein denaturation during manipulation. To address such problems, carriers act as a vehicle loaded with high concentrations of therapeutics, providing simultaneously a protective environment, either for local or systemic delivery, thus increasing the drug lifetime. The use of protein cages of non-viral origin as nanomaterials for biomedicine and biotechnology provides a number of unique advantages over other types of vehicles for drug delivery. Their biological origin makes them both amenable to genetic modification and large-scale production. Genetic modification enables the site-specific introduction of chemical and/or structural functionality onto highly symmetric protein cage platforms. By either chemical and/or genetic subunit alterations, it is feasible to simultaneously add new functions to different particle surfaces to direct cage assembly, encapsulation of a synthetic cargo, or targeting to a specific surface or cell. Regions not directly involved in vehicle assembly are generally more suitable to such modifications without losing the desired cage-like architecture. On the other hand, studies like those performed by Sutter and Seebeck pinpoint cargo protein features necessary for its encapsulation, paving the way for future development of protein-based nano-compartments for several nanotechnological applications. The discovery and understanding of signal sequences able to direct enzyme encapsulation into BMC and the underlying mechanisms of such process are key milestones in our understanding of BMC assembly, and leads the way for the development of these bacterial organelles toward biotechnological and biomedical applications. The simplicity of this system makes it attractive for engineering studies aimed at encapsulating distinct enzymes in order to create new vehicles to encapsulate, target and deliver therapeutic cargoes. There is still a long way ahead to explore in this area, but one can envision a great potential for $\mathrm{BMC}$ as drug delivery systems. Nowadays, the pharmaceutical industry is having trouble in discovering new drugs to treat a wide variety of diseases. This problem is being a serious limitation in conditions like cancer. Many of the substances that have proven to be highly 
efficient in removing tumor cells have been lately discarded due to its high toxicity or lack of specificity. The possibility of having a vehicle providing such specificity, by binding to a specific receptor, opens a wide range of possibilities for the rescue of these substances of low specificity.

The use of protein-based architectures is an exciting and fruitful scenario in the design of nano-cages for drug delivery, based on both protein engineering and the use of microbial cell factories. And although the understanding and putative engineering of protein assembling have a long way ahead, novel principles and promising strategies of protein manipulation point out the possibility of the rational construction of nanoscale protein cages as a viable concept.

\section{Acknowledgements}

The authors appreciate the financial support received for the design and microbial production of recombinant proteins for biomedical applications from MICINN (BFU2010-17450, ACI2009-0919, IT2009-0021, EUI2008-03610), AGAUR (2009SGR-108), MCYT (BIO2007-67904-C02-01), CIBER de Bioingeniería, Biomateriales y Nanomedicina (CIBER-BBN), Spain, and from the European Science Foundation, which is also funded by the European Commission, Contract no. ERAS-CT-2003-980409 of the Sixth Framework Programme (ERANET-IB 08-007). The authors also appreciate the financial support through the project "Development of nanomedicines for enzymatic replacement therapy in Fabry disease" granted by the Fundació Marató TV3.

\section{Author details}

${ }^{1}$ CIBER de Bioingeniería, Biomateriales y Nanomedicina (CIBER-BBN), Barcelona, Spain. ${ }^{2}$ Institute for Biotechnology and Biomedicine, Universitat Autònoma de Barcelona, Bellaterra, 08193 Barcelona, Spain. ${ }^{3}$ Laboratory of Inmunology, Regional Norte de la Universidad de la República, 50.000 Salto, Uruguay. ${ }^{4}$ Department of Genetics and Microbiology, Universitat Autònoma de Barcelona, Bellaterra, 08193 Barcelona, Spain.

\section{Authors' contributions}

JLC and JC have equally contributed to this work. Both authors read and approved the final manuscript.

\section{Competing interests}

The authors declare that they have no competing interests.

Received: 2 August 2011 Accepted: 3 November 2011

Published: 3 November 2011

\section{References}

1. Bobik TA: Polyhedral organelles compartmenting bacterial metabolic processes. Appl Microbiol Biotechnol 2006, 70:517-525.

2. Cheng S, Liu Y, Crowley CS, Yeates TO, Bobik TA: Bacterial microcompartments: their properties and paradoxes. Bioessays 2008 30:1084-1095.

3. Gitai Z: The new bacterial cell biology: moving parts and subcellular architecture. Cell 2005, 120:577-586.

4. Rudner DZ, Losick R: Protein subcellular localization in bacteria. Cold Spring Harb Perspect Biol 2010, 2:a000307.

5. Shively JM, Decker GL, Greenawalt JW: Comparative ultrastructure of the thiobacilli. J Bacteriol 1970, 101:618-627.

6. Shively JM, Ball F, Brown DH, Saunders RE: Functional organelles in prokaryotes: polyhedral inclusions (carboxysomes) of Thiobacillus neapolitanus. Science 1973, 182:584-586.

7. Chen P, Andersson DI, Roth JR: The control region of the $\mathrm{pdu} / \mathrm{cob}$ regulon in Salmonella typhimurium. J Bacteriol 1994, 176:5474-5482.
8. Kofoid E, Rappleye C, Stojiljkovic I, Roth J: The 17-gene ethanolamine (eut) operon of Salmonella typhimurium encodes five homologues of carboxysome shell proteins. J Bacteriol 1999, 181:5317-5329.

9. Kerfeld CA, Sawaya MR, Tanaka S, Nguyen CV, Phillips M, Beeby M, Yeates TO: Protein structures forming the shell of primitive bacterial organelles. Science 2005, 309:936-938.

10. Cannon GC, Bradburne CE, Aldrich HC, Baker SH, Heinhorst S, Shively JM: Microcompartments in prokaryotes: carboxysomes and related polyhedra. Appl Environ Microbiol 2001, 67:5351-5361.

11. Kaplan A, Reinhold L: Co2 concentrating mechanisms in photosynthetic microorganisms. Annu Rev Plant Physiol Plant Mol Biol 1999, 50:539-570.

12. Shively JM, van KG, Meijer WG: Something from almost nothing: carbon dioxide fixation in chemoautotrophs. Annu Rev Microbiol 1998, 52:191-230.

13. Badger MR, Price GD: CO2 concentrating mechanisms in cyanobacteria: molecular components, their diversity and evolution. J Exp Bot 2003, 54:609-622.

14. McKay RM, Gibbs SP, Espie GS: Effect of dissolved inorganic carbon on the expression of carboxysomes, localization of Rubisco and the mode of inorganic carbon transport in cells of the cyanobacterium \&lt;i\&gt; Synechococcus\&lt;/i\&gt; UTEX 625. Archives of Microbiology 1993, 159:21-29

15. English RS, Jin S, Shively JM: Use of Electroporation To Generate a Thiobacillus neapolitanus Carboxysome Mutant. Appl Environ Microbiol 1995, 61:3256-3260.

16. Price GD, Badger MR: Isolation and Characterization of High CO(2)Requiring-Mutants of the Cyanobacterium Synechococcus PCC7942: Two Phenotypes that Accumulate Inorganic Carbon but Are Apparently Unable to Generate CO(2) within the Carboxysome. Plant Physiol 1989, 91:514-525.

17. Price GD, Coleman JR, Badger MR: Association of Carbonic Anhydrase Activity with Carboxysomes Isolated from the Cyanobacterium Synechococcus PCC7942. Plant Physiol 1992, 100:784-793.

18. So AK, Espie GS, Williams EB, Shively JM, Heinhorst S, Cannon GC: A novel evolutionary lineage of carbonic anhydrase (epsilon class) is a component of the carboxysome shell. J Bacteriol 2004, 186:623-630.

19. Yu JW, Price GD, Song L, Badger MR: Isolation of a Putative Carboxysomal Carbonic Anhydrase Gene from the Cyanobacterium Synechococcus PCC7942. Plant Physiol 1992, 100:794-800.

20. Bobik TA, Havemann GD, Busch RJ, Williams DS, Aldrich HC: The propanediol utilization (pdu) operon of Salmonella enterica serovar Typhimurium LT2 includes genes necessary for formation of polyhedral organelles involved in coenzyme B(12)-dependent 1, 2-propanediol degradation. J Bacteriol 1999, 181:5967-5975.

21. Havemann GD, Sampson EM, Bobik TA: PduA is a shell protein of polyhedral organelles involved in coenzyme B(12)-dependent degradation of 1,2-propanediol in Salmonella enterica serovar typhimurium LT2. J Bacteriol 2002, 184:1253-1261.

22. Havemann GD, Bobik TA: Protein content of polyhedral organelles involved in coenzyme B12-dependent degradation of 1,2-propanediol in Salmonella enterica serovar Typhimurium LT2. J Bacteriol 2003, 185:5086-5095.

23. Leal NA, Havemann GD, Bobik TA: PduP is a coenzyme-a-acylating propionaldehyde dehydrogenase associated with the polyhedral bodies involved in B12-dependent 1,2-propanediol degradation by Salmonella enterica serovar Typhimurium LT2. Arch Microbiol 2003, 180:353-361.

24. Rondon MR, Horswill AR, Escalante-Semerena JC: DNA polymerase I function is required for the utilization of ethanolamine, 1,2-propanediol, and propionate by Salmonella typhimurium LT2. J Bacteriol 1995, 177:7119-7124.

25. Rondon MR, Kazmierczak R, Escalante-Semerena JC: Glutathione is required for maximal transcription of the cobalamin biosynthetic and 1,2propanediol utilization (cob/pdu) regulon and for the catabolism of ethanolamine, 1,2-propanediol, and propionate in Salmonella typhimurium LT2. J Bacteriol 1995, 177:5434-5439.

26. Sampson EM, Bobik TA: Microcompartments for B12-dependent 1,2 propanediol degradation provide protection from DNA and cellular damage by a reactive metabolic intermediate. J Bacteriol 2008, 190:2966-2971

27. Penrod JT, Roth JR: Conserving a volatile metabolite: a role for carboxysome-like organelles in Salmonella enterica. J Bacterio/ 2006 188:2865-2874 
28. Roof DM, Roth JR: Ethanolamine utilization in Salmonella typhimurium. J Bacteriol 1988, 170:3855-3863.

29. Stojiljkovic I, Baumler AJ, Heffron F: Ethanolamine utilization in Salmonella typhimurium: nucleotide sequence, protein expression, and mutational analysis of the cchA cchB eutE eutJ eutG eutH gene cluster. J Bacteriol 1995, 177:1357-1366.

30. Brinsmade SR, Paldon T, Escalante-Semerena JC: Minimal functions and physiological conditions required for growth of salmonella enterica on ethanolamine in the absence of the metabolosome. J Bacteriol 2005, 187:8039-8046.

31. Yeates TO, Crowley CS, Tanaka S: Bacterial microcompartment organelles: protein shell structure and evolution. Annu Rev Biophys 2010, 39:185-205.

32. Tanaka S, Kerfeld CA, Sawaya MR, Cai F, Heinhorst S, Cannon GC, Yeates TO: Atomic-level models of the bacterial carboxysome shell. Science 2008, 319:1083-1086.

33. Tanaka S, Sawaya MR, Yeates TO: Structure and mechanisms of a proteinbased organelle in Escherichia coli. Science 2010, 327:81-84.

34. Parsons JB, Frank S, Bhella D, Liang M, Prentice MB, Mulvihill DP, Warren MJ: Synthesis of empty bacterial microcompartments, directed organelle protein incorporation, and evidence of filament-associated organelle movement. Mol Cell 2010, 38:305-315.

35. Cai F, Menon BB, Cannon GC, Curry KJ, Shively JM, Heinhorst S: The pentameric vertex proteins are necessary for the icosahedral carboxysome shell to function as a CO2 leakage barrier. PLoS One 2009, 4:e7521.

36. Langer R, Tirrell DA: Designing materials for biology and medicine. Nature 2004, 428:487-492

37. Lee KY, Yuk SH: Polymeric protein delivery systems. Progress in Polymer Science 2007, 32:669-697.

38. Pawar R, Ben-Ari A, Domb AJ: Protein and peptide parenteral controlled delivery. Expert Opin Biol Ther 2004, 4:1203-1212.

39. Shi Y, Li LC: Current advances in sustained-release systems for parenteral drug delivery. Expert Opin Drug Deliv 2005, 2:1039-1058.

40. Cho K, Wang X, Nie S, Chen ZG, Shin DM: Therapeutic nanoparticles for drug delivery in cancer. Clin Cancer Res 2008, 14:1310-1316.

41. Kingsley JD, Dou H, Morehead J, Rabinow B, Gendelman HE, Destache CJ: Nanotechnology: a focus on nanoparticles as a drug delivery system. $J$ Neuroimmune Pharmacol 2006, 1:340-350.

42. Singh R, Lillard JW Jr: Nanoparticle-based targeted drug delivery. Exp Mol Pathol 2009, 86:215-223.

43. Hans ML, Lowman AM: Biodegradable nanoparticles for drug delivery and targeting. Current Opinion in Solid State and Materials Science 2002, 6:319-327.

44. Vazquez $E$, Villaverde A: Engineering building blocks for self-assembling protein nanoparticles. Microb Cell Fact 2010, 9:101.

45. Gazit E: Self-assembled peptide nanostructures: the design of molecular building blocks and their technological utilization. Chem Soc Rev 2007, 36:1263-1269.

46. Tsai CJ, Zheng J, Zanuy D, Haspel N, Wolfson H, Aleman C, Nussinov R: Principles of nanostructure design with protein building blocks. Proteins 2007, 68:1-12.

47. Garcia-Fruitos E, Gonzalez-Montalban N, Morell M, Vera A, Ferraz RM, Aris A, Ventura S, Villaverde A: Aggregation as bacterial inclusion bodies does not imply inactivation of enzymes and fluorescent proteins. Microb Cell Fact 2005, 4:27.

48. Garcia-Fruitos E: Inclusion bodies: a new concept. Microb Cell Fact 2010, 9:80.

49. Wu W, Xing L, Zhou B, Lin Z: Active protein aggregates induced by terminally attached self-assembling peptide ELK16 in Escherichia coli. Microb Cell Fact 2011, 10:9.

50. Xing L, Wu W, Zhou B, Lin Z: Streamlined protein expression and purification using cleavable self-aggregating tags. Microb Cell Fact 2011, 10:42.

51. Vazquez E, Roldan M, ez-Gil C, Unzueta U, Domingo-Espin J, Cedano J, Conchillo O, Ratera I, Veciana J, Daura X, et al: Protein nanodisk assembling and intracellular trafficking powered by an arginine-rich (R9) peptide. Nanomedicine (Lond) 2010, 5:259-268.

52. Maham A, Tang Z, Wu H, Wang J, Lin Y: Protein-based nanomedicine platforms for drug delivery. Small 2009, 5:1706-1721.
53. Uchida $M$, Klem $M \Gamma$, Allen $M$, Suci P, Flenniken M, Gillitzer E, Varpness Z, Liepold $L \Gamma$, Young M, Douglas T: Biological Containers: Protein Cages as Multifunctional Nanoplatforms. Adv Mater 2007, 19:1025-1042.

54. Uchida M, Kang S, Reichhardt C, Harlen K, Douglas T: The ferritin superfamily: Supramolecular templates for materials synthesis. Biochim Biophys Acta 2010, 1800:834-845.

55. Douglas T, Young M: Viruses: making friends with old foes. Science 2006, 312:873-875

56. Cot SS, So AK, Espie GS: A multiprotein bicarbonate dehydration complex essential to carboxysome function in cyanobacteria. J Bacteriol 2008, 190:936-945

57. Long BM, Badger MR, Whitney SM, Price GD: Analysis of carboxysomes from Synechococcus PCC7942 reveals multiple Rubisco complexes with carboxysomal proteins CcmM and CcaA. J Biol Chem 2007, 282:29323-29335.

58. Price GD, Howitt SM, Harrison K, Badger MR: Analysis of a genomic DNA region from the cyanobacterium Synechococcus sp. strain PCC7942 involved in carboxysome assembly and function. J Bacteriol 1993, 175:2871-2879

59. Fan C, Cheng S, Liu Y, Escobar CM, Crowley CS, Jefferson RE, Yeates TO, Bobik TA: Short $\mathrm{N}$-terminal sequences package proteins into bacterial microcompartments. Proc Natl Acad Sci USA 2010, 107:7509-7514.

60. Menon BB, Dou Z, Heinhorst S, Shively JM, Cannon GC: Halothiobacillus neapolitanus carboxysomes sequester heterologous and chimeric RubisCO species. PLoS One 2008, 3:e3570.

61. Sutter M, Boehringer D, Gutmann S, Gunther S, Prangishvili D, Loessner MJ, Stetter KO, Weber-Ban E, Ban N: Structural basis of enzyme encapsulation into a bacterial nanocompartment. Nat Struct Mol Biol 2008, 15:939-947.

62. Valdes-Stauber N, Scherer S: Isolation and characterization of Linocin M18, a bacteriocin produced by Brevibacterium linens. Appl Environ Microbiol 1994, 60:3809-3814.

63. Seebeck FP, Woycechowsky K, Zhuang W, Rabe JP, Hilvert D: A simple tagging system for protein encapsulation. J Am Chem Soc 2006, 128:4516-4517.

64. Pasqualini R, Ruoslahti E: Tissue targeting with phage peptide libraries1. Mol Psychiatry 1996, 1:423.

65. Pasqualini R, Ruoslahti E: Organ targeting in vivo using phage display peptide libraries. Nature 1996, 380:364-366.

66. Flenniken ML, Willits DA, Harmsen AL, Liepold LO, Harmsen AG, Young MJ, Douglas T: Melanoma and lymphocyte cell-specific targeting incorporated into a heat shock protein cage architecture. Chem Biol 2006, 13:161-170.

67. Christie RJ, Grainger DW: Design strategies to improve soluble macromolecular delivery constructs. Adv Drug Deliv Rev 2003, 55:421-437.

68. Dalmau M, Lim S, Wang SW: Design of a pH-dependent molecular switch in a caged protein platform. Nano Lett 2009, 9:160-166.

69. Dalmau M, Lim S, Wang SW: pH-triggered disassembly in a caged protein complex. Biomacromolecules 2009, 10:3199-3206.

70. Veronese FM, Pasut G: PEGylation, successful approach to drug delivery. Drug Discov Today 2005, 10:1451-1458.

\section{doi:10.1186/1475-2859-10-92}

Cite this article as: Corchero and Cedano: Self-assembling, proteinbased intracellular bacterial organelles: emerging vehicles for encapsulating, targeting and delivering therapeutical cargoes. Microbial Cell Factories 2011 10:92. 